\title{
Metas académicas de los estudiantes de secundaria relacionadas con la variable género*
}

\author{
Jorge Enrique Díaz Pinzón ${ }^{1}$
}

Recibido: 26-11-2019

Aceptado: 06-05-2020

\section{Resumen}

Esta investigación tuvo como objetivo identificar la relación entre el género de los estudiantes de básica secundaria y media vocacional y su perspectiva sobre sus metas académicas, logro y refuerzo social. Se realizó una encuesta a 20 estudiantes de la I. E. General Santander, con edades entre 12 y 18 años, aplicando un instrumento de 20 preguntas con escala de tipo Likert. El resultado demostró que el valor de significancia de todas las preguntas es mayor a $\alpha$ = 0,05; por lo tanto, se acepta la hipótesis nula, es decir, que no hay diferencias en el promedio de las respuestas obtenido por los estudiantes de grado $7 .^{\circ}, 8 .^{\circ}$ y $10 .^{\circ}$ según el sexo. Se concluyó que la variable sexo no muestra grandes diferencias en ninguno de los tres factores analizados, y que las metas de aprendizaje sí tienen mayor relevancia frente a metas de logro y refuerzo social.

Palabras clave: motivación académica, metas académicas, metas de aprendizaje, metas de rendimiento, metas sociales, rendimiento académico.

\footnotetext{
* Artículo resultado de investigación. Hace parte de la investigación titulada: Metas académicas de los estudiantes de secundaria relacionada con la variable sexo, desarrollada desde marzo de 2019 hasta agosto de 2019 en el municipio de Soacha, Cundinamarca, Colombia.

1. Magíster en Gestión de la Tecnología Educativa, especialista en Administración de la Informática Educativa, ingeniero agrónomo. Docente titular de Matemáticas e investigador de la I. E. General Santander, Soacha, Colombia.

Correo electrónico: jediazp@unal.edu.co/jorgediaz333@gmail.com

ORCID: https://orcid.org/0000-0002-8870-7769

Google Scholar: https://scholar.google.es/citations?user=f4PqfaoAAAAJ\&hl=es
} 


\section{Academic goals of high school students related to the gender variable}

\section{Abstract}

This research aimed to identify the relationship between the gender of elementary school and vocational high school students and their perspective on their academic goals, achievement and social reinforcement. A survey was carried out on 20 students of the General Santander School, aged between 12 and 18 years, applying an instrument of 20 questions with a Likert-type scale. The result showed that the significance value of all the questions is greater than $\alpha=0.05$; Therefore, the null hypothesis is accepted, that is, there are no differences in the average of the responses obtained by the 7th, 8th and 10th grade students according to sex. It was concluded that the gender variable does not show great differences in any of the three factors analyzed, and that learning goals do have greater relevance compared to goals of achievement and social reinforcement.

Keywords: academic motivation, academic goals, learning goals, performance goals, social goals, academic performance.

\section{Introducción}

Nuestro cometido y vocación como docentes se cimienta en el ejercicio diario de la enseñanza y el aprendizaje que busca materializar el discurso de la educación. En tal ejercicio, se resaltan tres elementos fundamentales: el niño, el aprendizaje, y el contexto que le rodea lo que debe aprender para dedicarse como individuo dentro de esa sociedad, como describe Avendaño (2013). 
Son varios los factores motivacionales que tienen los estudiantes, como refiere Moneo (2017, citado por Rodríguez y Avendaño, 2018), entre los cuales alude la meta como el objetivo intelectual que se propone lograr, el motivo o deseo de alcanzar la meta, las expectativas que se forjan —como las creencias subjetivas de alcanzar o no la meta planteadas-, los planes de acción y las acciones orientadas conseguir los logros (considerados en términos de éxito o frustración), la autonomía, la gratitud de las actividades y los logros alcanzados, el trabajo en grupo y la aprobación de los mismos. Estos y otros factores motivacionales son los que el estudiante ejerce y consigue desarrollar durante su vida formativa.

Al momento de buscar las causas de la frustración escolar, la atención se dirige hacia los programas de estudio, la masificación de las aulas, la falta de recursos de las instituciones y muchas veces al papel de los padres cuya actitud insinúa que su responsabilidad termina donde empieza la de los maestros (Navarro, 2003). Uno de los problemas que enfrenta la educación en el mundo es el mejoramiento de los sistemas educativos. Un factor importante para ello son las metas de aprendizaje y cómo estas repercuten en las valoraciones y resultados académicos (Pérez y Castejón, 1997; Piñeros y Rodríguez, 1998; Cepeda y Caicedo, 2007) o cómo uno influye en el otro. Las diferentes investigaciones que se han realizado en Latinoamérica y en el mundo apuntan a determinar qué factores son claves, pero en este aspecto todavía no hay resultados concluyentes. Para este caso la muestra se formó en la Institución Educativa General Santander del municipio de Soacha (Cundinamarca, Colombia). El éxito escolar, de acuerdo con la disertación de Redondo (1997), demanda de un alto grado de adhesión a los resultados, los medios y los valores de la institución educativa, que posiblemente no todos los estudiantes presentan.

Los modelos normativos de metas sostienen que los estudiantes pueden ser clasificados según el tipo de meta que ocupen y establecen al menos dos tipos: sociales y académicas. Las metas sociales se definen por razones de orden social que los estudiantes pueden tener para conducirse en la situación académica (sentirse miembros de un grupo, ayudar o recibir ayuda de otros, tener 
amigos, tratarse con lo demás, etc.) (Wentzel, 1996; Wentzel, 1998; Wentzel y Wigfield, 1998, citados por Navas, Iborra y Sampascual, 2007). Y las metas académicas apuntan a los motivos de orden académico que tienen los estudiantes para guiar su comportamiento en el aula: dominar o realizar la tarea, señalar su habilidad y tener buen rendimiento y buenos resultados (Ames, 1992; Pintrich, 2000; Pintrich y Shunk, 1996; Urdan, 1997, citados por Navas et al., 2007).

Dentro del acercamiento a las metas académicas, las investigaciones de Dweck y Elliot (1983) y Dweck (1986) se concentraron, inicialmente, en el patrón de proceder de logro adaptativo de algunos sujetos y en el de los que manifiestan indefensión ante los obstáculos que se les presentan. Se refieren dos tipos de conducta: por un lado, la de los sujetos que parecen disfrutar con los retos o los desafíos que se originan ante ellos y permanecen en el aprendizaje y, por otro, el comportamiento que presentan los sujetos que, ante potenciales desafíos o nuevos retos, exponen una conducta de evitación y de baja permanencia en la realización de la tarea propuesta, prestando más atención a la experiencia negativa que han logrado tener en situaciones análogas de desafío y a la baja autoestima que les produce tener que enfrentar estos obstáculos.

En conexión con esto, se consideró, inicialmente, la existencia de dos tipos de metas: de aprendizaje y de ejecución. Las primeras se definen por el interés por la adquisición y el desarrollo de nuevas capacidades y conocimientos; estas orientan al sujeto hacia la tarea de aprendizaje y hacia una búsqueda de lo que presuma un reto. Las segundas se caracterizan por el interés por conseguir juicios y valoraciones positivas sobre la propia cabida y están relacionadas con un patrón motivacional de abandono.

Al confrontar las definiciones que brindan varios autores en la conceptualización de la orientación general a metas de logro, se propone que todas ellas concuerdan en : 1) que las metas representan un conjunto de pensamientos y razones para proceder, 2) que reflejan el deseo de manifestar la propia valía y la competen- 
cia en una actividad explícita, y 3) que intervienen en el modo en el que los estudiantes se acercan a sus tareas académicas (Jover y Sampascual, 2008).

\section{Metodología}

Siguiendo a Rodríguez y Avendaño (2018), el diseño metodológico que se utiliza como ruta para conseguir el objetivo propuesto es de tipo no experimental que detalla la dinámica de una realidad, bajo la modalidad transaccional correlacional de la variable género. Además, se tuvo en cuenta como variable adicional el contexto en el que se ubica cada una de las muestras poblacionales.

Dada la necesidad de conocer la relación entre las metas de aprendizaje de los estudiantes de secundaria y su sexo, se requiere recoger información objetiva a partir de una muestra de la población de la I. E. General Santander. Para tal fin se ha tomado una muestra aleatoria de cuatro estudiantes por grado entre $7 .^{\circ}, 8 .^{\circ} \mathrm{y}$ $10 .^{\circ}$ con un total de $n=20$. Los estudiantes pertenecen a la misma sede y jornada (tarde), es decir se encuentran dentro del mismo contexto educativo. Ahora como sistema de recopilación de datos, se realizó una encuesta aplicando un instrumento de 20 preguntas con escala tipo Likert (ver tabla 1).

La escala tipo Likert es una herramienta de medición o recolección de datos cuantitativos utilizado dentro de la investigación. Es un tipo de escala agregada que corresponde a un nivel de medición ordinal; se compone de una serie de ítems o juicios a modo de afirmaciones ante los cuales se requiere la reacción del sujeto. El estímulo (ítem o juicio) que se presenta al sujeto, personifica la propiedad que el investigador está interesado en medir y las respuestas son requeridas en términos de grados de acuerdo o desacuerdo que el sujeto tenga con el dictamen en particular. Son cinco el número de opciones de respuesta más usado, donde a cada categoría se le atribuye un valor numérico que llevará al sujeto a una puntuación total producto de las puntuaciones de todos los 
ítems. Dicha puntuación final indica la posición del sujeto dentro de la escala (Méndez y Peña, 2007).

Tabla 1. Encuesta escala tipo Likert.

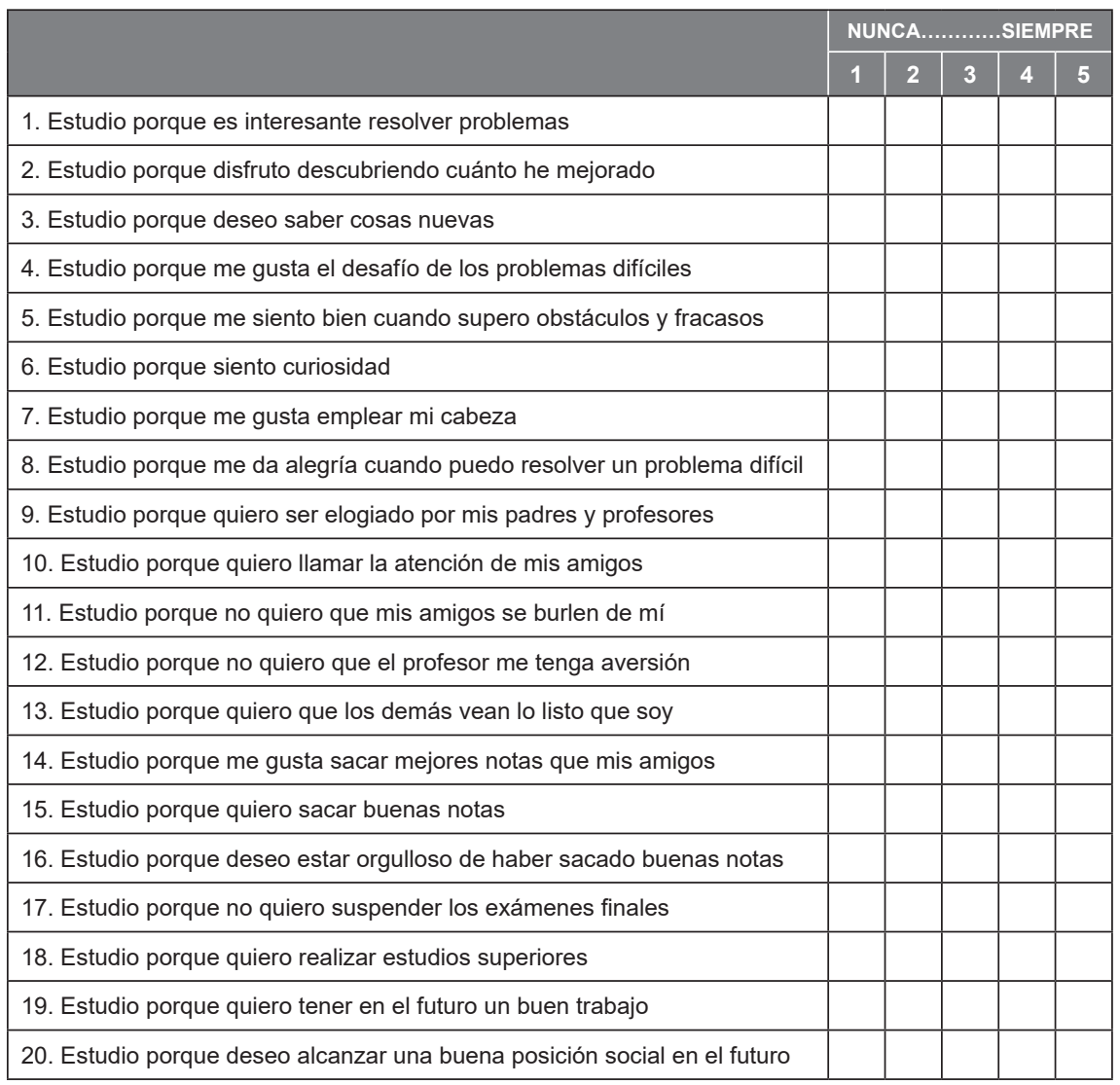

Fuente: elaboración propia

La información de las encuestas ha sido tabulada en el programa SPPS v. 20, generando una matriz de datos que corresponden a los tres factores, a saber: el factor I agrupa los ítems 1-8, sobre metas de aprendizaje; el factor II incluye los ítems 9 y 15-20, relacionados con metas de logro; finalmente, el factor III reúne los ítems 10 a 14, acerca de metas de refuerzo social. Para los distintos grupos de resultados, se realizó la sumatoria de las respuestas de cada estudiante, para luego proceder a su análisis estadístico. 
Para obtener información relevante acerca de la tendencia de los estudiantes de ambos sexos a nivel de secundaria sobre sus metas académicas, debe contarse con una muestra representativa. En el caso presente, partiendo de una muestra mixta de 20 estudiantes (10 mujeres y 10 hombres), se busca medir el comportamiento de dos poblaciones relacionadas haciendo uso de la prueba "t de estudiante", para muestras independientes.

Hipótesis alterna ( $\mathrm{Ha}$ ): Hay diferencias en el promedio de las respuestas obtenidas, según el sexo de los estudiantes de grado $7 .^{\circ}$, $8 .^{\circ}$ y $10 .^{\circ}$ de la jornada tarde de la Institución Educativa General Santander del municipio de Soacha (Cundinamarca).

Hipótesis nula (Ho): No hay diferencias en el promedio de las respuestas obtenidas, según el sexo de los estudiantes de grado $7 .^{\circ}$, $8 .^{\circ}$ y $10 .^{\circ}$ de la jornada tarde de la Institución Educativa General Santander del municipio de Soacha (Cundinamarca).

Prueba estadística: el valor de significancia de la prueba es de $\alpha=$ 0.05 (5\%). Si es mayor, se acepta la hipótesis nula; si es menor, se rechaza la hipótesis nula. Para la comparación entre medias independientes, se utilizó el Software SPSS v 20.

Para realizar el ejercicio, se seleccionó de manera aleatoria a 10 estudiantes de sexo masculino y 10 de sexo femenino de los grados $7 .^{\circ}, 8 .^{\circ}$ y $10 .^{\circ}$. La aplicación de la encuesta se realizó bajo la supervisión del docente que realizó está investigación,. Se les solicitó diligenciar la encuesta, sin límite de tiempo, la cual se recogió al terminar.

Para llevar a cabo la actividad, se empleó una encuesta de 20 preguntas con escala de respuestas tipo Likert, con cinco opciones de respuesta; el tiempo promedio de respuesta del cuestionario fue de cinco minutos. En el encabezado se indicaba por escrito que la puntuación de 1 correspondía a la situación en la cual el comportamiento descrito nunca se presentaba. Contrariamente, la puntuación de 5 correspondía a la manifestación constante del comportamiento, indicado con la palabra siempre. 
Los datos personales de cada estudiante aparecían en el encabezado de la encuesta. Allí se incluyó la edad, el sexo y sus resultados en matemáticas y lenguaje.

Los estudiantes encuestados fueron reunidos por nivel educativo en un espacio diferente (aula de clase). Después de hacerles una breve inducción acerca de la naturaleza de la encuesta, cada estudiante de manera individual respondió a las diferentes preguntas formuladas. Una vez diligenciadas las encuestas, se procedió a su tabulación y análisis estadístico.

\section{Análisis estadístico}

La media de edad para las participantes de sexo femenino fue de $M=14,55$ años; para esta población, la media en lenguaje fe de $M=7,2$ y la media en matemáticas, $M=7,2$. La media para los participantes de sexo masculino fue de $M=15,21$ años; para esta población, la media en lenguaje fue de $M=6,9$ y la media en matemáticas, $M=6,9$. En la tabla 2 se puede apreciar los resultados de la encuesta.

Tabla 2. Resultados de la encuesta por sexo del estudiante.

\begin{tabular}{|l|l|l|l|l|l|l|l|l|l|l|l|l|l|l|l|l|l|l|l|c|}
\hline 1 & 2 & 3 & 4 & 5 & 6 & 7 & 8 & 10 & 11 & 12 & 13 & 14 & 9 & 15 & 16 & 17 & 18 & 19 & 20 & SEXO \\
\hline 4 & 5 & 5 & 5 & 4 & 3 & 3 & 3 & 2 & 2 & 3 & 2 & 3 & 2 & 3 & 3 & 4 & 4 & 5 & 5 & Femenino \\
\hline 3 & 5 & 5 & 3 & 5 & 5 & 4 & 3 & 2 & 1 & 1 & 3 & 1 & 4 & 4 & 4 & 4 & 5 & 5 & 5 & Femenino \\
\hline 2 & 4 & 4 & 3 & 4 & 5 & 3 & 2 & 1 & 1 & 1 & 1 & 3 & 4 & 2 & 4 & 4 & 3 & 3 & 3 & Femenino \\
\hline 3 & 4 & 5 & 3 & 5 & 4 & 4 & 5 & 1 & 1 & 2 & 4 & 3 & 5 & 5 & 5 & 4 & 5 & 5 & 5 & Femenino \\
\hline 3 & 4 & 5 & 4 & 4 & 5 & 3 & 5 & 1 & 1 & 1 & 3 & 1 & 4 & 3 & 5 & 2 & 5 & 5 & 4 & Femenino \\
\hline 3 & 4 & 3 & 3 & 4 & 3 & 3 & 4 & 2 & 2 & 2 & 3 & 3 & 3 & 4 & 4 & 3 & 4 & 5 & 4 & Femenino \\
\hline 3 & 5 & 4 & 4 & 5 & 4 & 4 & 5 & 1 & 1 & 1 & 2 & 2 & 5 & 3 & 5 & 4 & 5 & 5 & 5 & Femenino \\
\hline 2 & 5 & 3 & 2 & 5 & 5 & 5 & 3 & 1 & 1 & 2 & 4 & 5 & 5 & 5 & 3 & 5 & 5 & 5 & 5 & Femenino \\
\hline 3 & 4 & 5 & 3 & 4 & 3 & 4 & 5 & 2 & 1 & 1 & 3 & 3 & 5 & 5 & 5 & 5 & 5 & 5 & 4 & Femenino \\
\hline 5 & 5 & 5 & 5 & 5 & 1 & 5 & 5 & 1 & 1 & 1 & 5 & 1 & 4 & 5 & 5 & 4 & 5 & 5 & 5 & Femenino \\
\hline 4 & 5 & 5 & 3 & 4 & 1 & 3 & 4 & 1 & 1 & 2 & 3 & 3 & 5 & 5 & 5 & 4 & 5 & 5 & 5 & Masculino \\
\hline 4 & 5 & 5 & 5 & 3 & 5 & 5 & 5 & 3 & 5 & 3 & 4 & 5 & 4 & 5 & 5 & 5 & 4 & 3 & 5 & Masculino
\end{tabular}




\begin{tabular}{|l|l|l|l|l|l|l|l|l|l|l|l|l|l|l|l|l|l|l|l|l|}
\hline 1 & 2 & 3 & 4 & 5 & 6 & 7 & 8 & 10 & 11 & 12 & 13 & 14 & 9 & 15 & 16 & 17 & 18 & 19 & 20 & SEXO \\
\hline 4 & 3 & 4 & 4 & 5 & 4 & 4 & 5 & 2 & 1 & 2 & 3 & 3 & 4 & 4 & 4 & 4 & 5 & 5 & 5 & Masculino \\
\hline 5 & 4 & 5 & 4 & 5 & 5 & 4 & 4 & 4 & 1 & 1 & 3 & 3 & 5 & 4 & 5 & 5 & 5 & 5 & 5 & Masculino \\
\hline 4 & 4 & 5 & 3 & 5 & 4 & 3 & 4 & 1 & 1 & 2 & 3 & 3 & 3 & 1 & 5 & 3 & 5 & 5 & 4 & Masculino \\
\hline 2 & 4 & 3 & 1 & 5 & 2 & 3 & 4 & 1 & 2 & 1 & 2 & 2 & 4 & 5 & 4 & 1 & 3 & 5 & 5 & Masculino \\
\hline 4 & 5 & 5 & 2 & 4 & 3 & 1 & 5 & 3 & 2 & 3 & 4 & 3 & 4 & 2 & 4 & 3 & 4 & 5 & 4 & Masculino \\
\hline 4 & 4 & 5 & 4 & 4 & 4 & 5 & 3 & 1 & 1 & 1 & 2 & 2 & 3 & 4 & 4 & 3 & 4 & 4 & 4 & Masculino \\
\hline 4 & 5 & 5 & 3 & 5 & 5 & 5 & 4 & 1 & 4 & 1 & 4 & 4 & 5 & 3 & 5 & 4 & 5 & 5 & 5 & Masculino \\
\hline 3 & 4 & 5 & 2 & 4 & 2 & 2 & 4 & 5 & 4 & 4 & 2 & 5 & 5 & 5 & 5 & 3 & 4 & 5 & 5 & Masculino \\
\hline
\end{tabular}

Fuente: elaboración propia

En la tabla 3, se observa gran similitud en las medidas de tendencia central para las respuestas de ambos sexos referentes al factor I. Esto hace pensar que tanto los hombres como las mujeres tienen un claro interés por sus procesos de desarrollo personal.

En lo relativo al factor II, podría concluirse que mujeres y hombres tienen las mismas preocupaciones por su futuro. En cuanto al factor III, puede afirmarse que hay un promedio igual en los resultados de los hombres y las mujeres, lo cual supone igual interés por la opinión que otras personas puedan tener sobre su desempeño académico.

Tabla 3. Media, mediana y moda por factor.

\begin{tabular}{|l|l|c|c|}
\hline Factor I Femenino & \multicolumn{1}{|c|}{ Factor I Masculino } & \\
\hline Media & 3,9 & Media & 4 \\
\hline Mediana & 4 & Mediana & 4 \\
\hline Moda & 5 & Moda & 4 \\
\hline Factor II Femenino & & Factor II Masculino & \\
\hline Media & 2 & Media & 2,5 \\
\hline Mediana & 2 & Mediana & 2,5 \\
\hline Moda & 1 & Moda & 1 \\
\hline Factor III Femenino & & Media & 4,3 \\
\hline Media & 4,3 & Mediana & 5 \\
\hline Mediana & 5 & Moda & 5 \\
\hline Moda & 5 & & \\
\hline
\end{tabular}

Fuente: elaboración propia 


\section{Resultados}

Según los resultados de la muestra $n=20$, las diferencias entre hombres y mujeres con relación a los resultados de la encuesta no son significativas. No hay una diferencia de género, si se observa que la I. E. General Santander está ubicada en el área urbana, en donde los roles son similares tanto para jóvenes como para adultos - teniendo en cuenta que las medias en edad para las dos muestras son: femenino de $M=15$ años y masculino de $M=15,2$ años-. Puede que la muestra no sea cotejada al participar estudiantes de ambos sexos de los grados $7 .^{\circ}, 8 .^{\circ}$ y $10 .^{\circ}$.

En la tabla 4, se puede apreciar la anova (análisis de varianza, por sus siglas en inglés) con un factor, relacionada con el factor sexo y las 20 preguntas de la encuesta. Los grados de libertad se determinan según el número de observaciones en la muestra. En esta investigación se aprecia que los grados de libertad para cada pregunta son iguales (19), ya que el total de preguntas fueron 20. Ahora la prueba estadística " $F$ " de Fisher puede medir la significancia global del modelo, es decir si el modelo de regresión múltiple es estadísticamente significativo o no.

Se observa además el valor p para determinar si la diferencia en las desviaciones estándar o varianzas de las poblaciones es estadísticamente significativa (Sig). Para establecer si la diferencia entre las desviaciones estándar o las varianzas de las poblaciones es estadísticamente significativa, se compara el valor $p$ con el nivel de significancia. Por lo general, un nivel de significancia (denotado como $\alpha$ o alfa) de 0.05 funciona apropiadamente. Un nivel de significancia de 0.05 indica un riesgo de $5 \%$ de concluir que existe una diferencia cuando no hay una diferencia real (Minitab, s. f.). 
Tabla 4. Anova de un factor.

\begin{tabular}{|l|c|c|c|}
\hline & gl & F & Sig. \\
\hline Estudio porque es interesante resolver problemas & 19 &, 161 &, 983 \\
\hline Estudio porque disfruto descubriendo cuánto he mejorado & 19 &, 396 &, 869 \\
\hline Estudio porque deseo saber cosas nuevas & 19 &, 353 &, 896 \\
\hline Estudio porque me gusta el desafío de los problemas difíciles & 19 &, 855 &, 551 \\
\hline Estudio porque me siento bien cuando supero obstáculos y fracasos & 19 & 1,06 &, 433 \\
\hline Estudio porque siento curiosidad & 19 &, 580 &, 740 \\
\hline Estudio porque me gusta emplear mi cabeza & 19 & 2,14 &, 117 \\
\hline Estudio porque me da alegría cuando puedo resolver un problema difícil & 19 &, 501 &, 797 \\
\hline Estudio porque quiero ser elogiado por mis padres y profesores & 19 &, 516 &, 786 \\
\hline Estudio porque quiero llamar la atención de mis amigos & 19 &, 798 &, 588 \\
\hline Estudio porque no quiero que mis amigos se burlen de mí & 19 &, 443 &, 838 \\
\hline Estudio porque no quiero que el profesor me tenga aversión & 19 & 1,59 &, 227 \\
\hline Estudio porque quiero que los demás vean lo listo que soy & 19 &, 687 &, 664 \\
\hline Estudio porque me gusta sacar mejores notas que mis amigos & 19 & 19 & 19 \\
\hline Estudio porque quiero sacar buenas notas & 19 &, 560 \\
\hline Estudio porque deseo estar orgulloso de haber sacado buenas notas &, 283 &,, 935 \\
\hline Estudio porque no quiero suspender los exámenes finales & 19 &, 477 &, 366 \\
\hline Estudio porque quiero realizar estudios superiores & 19 & 1,44 &, 272 \\
\hline Estudio porque quiero tener en el futuro un buen trabajo & 19 \\
\hline Estudio porque deseo alcanzar una buena posición social en el futuro & 19 \\
\hline
\end{tabular}

Fuente: elaboración propia

Según el valor de significancia de la prueba es de $\alpha=0,05$ (5 $\%)$, si es mayor se acepta la hipótesis nula. En esta investigación se puede observar en la tabla 4, que el valor de significancia de todas las preguntas es mayor a $\alpha=0,05$, por lo tanto se acepta la hipótesis nula, que no hay diferencias en el promedio de las respuestas obtenido por los estudiantes de grado $7 .{ }^{\circ}, 8^{\circ}$ y $10 .^{\circ}$, según el sexo, de la jornada tarde de la Institución Educativa General Santander del municipio de Soacha (Cundinamarca).

En la figura 1, se observan los promedios por pregunta. Analizamos que las preguntas 10,11 y 12 son las de menor promedio, con 1,$8 ; 1,7$ y 1,75 respectivamente. Estos ítems afirmaban que "estudio porque quiero llamar la atención de mis amigos", "estudio porque 
no quiero que mis amigos se burlen de mí" y "estudio porque no quiero que el profesor me tenga aversión".

Los promedios más altos los encontramos en las respuestas 17 , 18, 19 y 20, con promedios de 4,5;4,75; 4,75 y 4,6 respectivamente. Estos ítems indicaban que "estudio porque no quiero suspender los exámenes finales", "estudio porque quiero realizar estudios superiores", "estudio porque quiero tener en el futuro un buen trabajo" y "estudio porque deseo alcanzar una buena posición social en el futuro".

Figura 1. Promedios por preguntas de la encuesta.

Promedios por pregunta

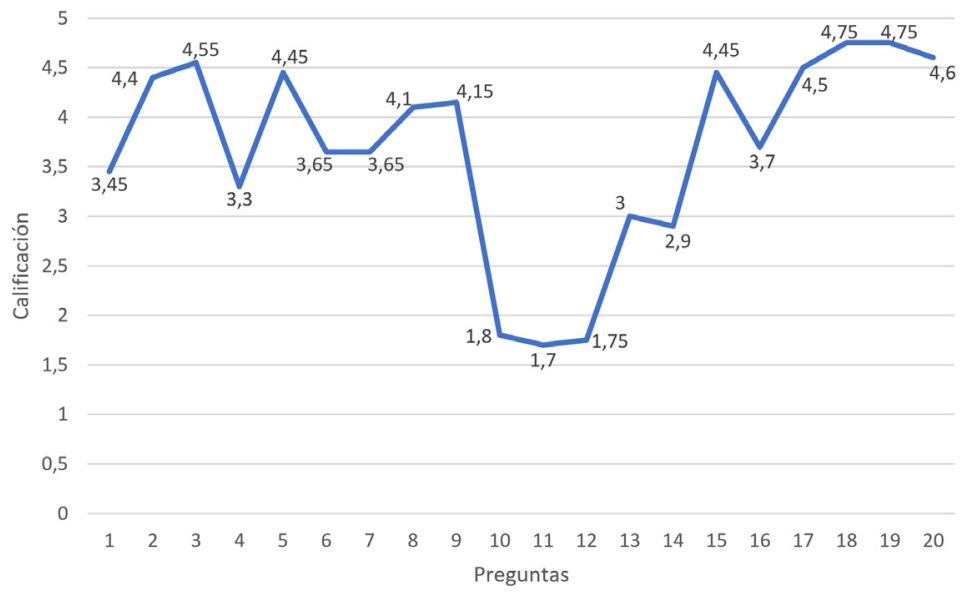

Fuente: elaboración propia

\section{Discusión de resultados}

Según Barca, Peralbo, Porto, Marcos y Brenilla (2011), en concreto, los datos indican que son las metas de aprendizaje y las metas de rendimiento/logro las que tienen una ocurrencia significativa en el grupo (los fines académicos del alumnado de educación secunda- 
ria obligatoria (ESO) y bachillerato con alto y bajo rendimiento escolar). Esto significa que el rendimiento que obtiene el estudiante tiende a ser más alto en la medida en que también es alta su opción por las metas de aprendizaje que, como ya se ha repetido, se dirigen a la motivación taxativa, una implicación clara en las tareas y el deseo de acrecentar la competencia.

En ese mismo plano Cornejo y Redondo (2007), aprecian la necesidad de examinar el aparente de causalidad lineal entre los factores escolares y los resultados educativos que están tácitos en la tradición de la eficacia escolar. La figura de características de las escuelas eficaces dice poco acerca de cómo se ajustan estos factores y, sobre todo, cómo es que estos se alcanzan a componer en la cultura escolar propia de la institución.

A esta afinidad resulta significativo el típico tópico de los padres que se aproximan al colegio a preguntar cómo va su hijo en las asignaturas y que no proyectan posibilidades de lo que este pueda hacer una vez culmine la enseñanza obligatoria. Parece como si todo el horizonte parental estuviese situado en el presente, y que fueran inexpertos para presagiar un camino futuro para el hijo (Pérez y Castejón, 1997).

Asimismo, en el estudio realizado por Navas et al. (2007), se planteaba si existían diferencias en las metas de los estudiantes que cursan Música como asignatura obligatoria y los que la cursan como voluntaria. Una vez analizados los datos comprobó que los estudiantes que estudian el área de Música como asignatura obligatoria necesariamente tienen una puntuación superior en metas de aprendizaje y de refuerzo social que los estudiantes que estudian esta área de forma optativa. Estos resultados son, cuando menos, incautos, puesto que llama la atención que se obtenga una puntuación superior en las metas académicas cuando el área es asignada $y$, sin embargo, sea inferior en el caso de la optatividad, donde el estudiante puede preferir el área de Música entre otras cinco áreas más. 


\section{Conclusiones}

Como conclusión, se puede decir que los factores del rendimiento académico no son predictores de éxito académico; faltan herramientas en el tratamiento de los datos para afirmar una u otra cosa. Además, se observó que la variable sexo no marca grandes diferencias en ninguno de los tres factores analizados. También se concluye que las metas de aprendizaje sí tienen una relevancia frente a metas de logro y refuerzo social.

Siguiendo el estudio de Rodríguez y Avendaño (2018), para el factor I (que acata a las metas de aprendizaje) se observa que la adquisición de conocimiento al enlazarlo con las ciencias naturales privilegia la competencia de la exploración y la curiosidad científica, obteniendo la aproximación creciente al conocimiento científico a partir del conocimiento y reconocimiento del medio natural (siendo el sexo masculino el que prevalece en esta intención). Para el segundo factor que incumbe a las metas de logro, el sexo femenino aviva mayor interés, prevaleciendo la meta contigua de obtener buenos resultados académicos. Esto, desde la perspectiva del estudiante, desemboca en un postura laboral y se establece una relación entre el saber y el hacer, que cimienta la causalidad de las ciencias para contribuir en la formación de hombres y mujeres como miembros activos de una sociedad. Entretanto, para el factor III, en el que se describen las metas de refuerzo o de reconocimiento social, para ambos sexos es insensible un reconocimiento, aunque en el sexo masculino prevalece la intención de la competencia para ser el mejor cotejado con su par académico; esto es característico no solo en las ciencias naturales, sino también en las demás áreas del conocimiento pues es intrínseco de la naturaleza humana.

En este estudio se reafirmó que al observar la tendencia en tres contextos variados, la cual es muy similar, se puede deducir que una circunstancia extrínseca como el medio social no es un factor de motivación que prima en un educando (Rodríguez y Avendaño, 2018). 
Esta investigación determinó también que los factores que prevalecen en la subjetividad de un educando y que lo motivan a plantearse una meta académica están encaminados al logro, ya sea por la pasión que le brinda solucionar un problema difícil (más sesgado hacia el sexo masculino), o el tener la perspectiva de un futuro mejor (que lo evidencia en mayor proporción el sexo femenino).

En esta misma línea incide el trabajo realizado por Barca et al. (2011), en el que se observa que el estudiante busca una indudable independencia en su proceso de estudio, desea conseguir competencias, perfeccionar sus conocimientos y dominar los desiguales contenidos de las materias. De aquí se desglosa que el profesorado convendrá tener en cuenta este tipo de metas imperiosas en el estudiante, de modo que beneficiará el aprendizaje, interesándose por el estudiante, renovando los contenidos, mostrando recursos instruccionales que involucren al estudiante en la aportación activa y participada del aprendizaje con los demás compañeros en el aula de clase.

Otra mención al respecto lo ofrecen Cepeda y Caicedo (2007), quienes sugieren que la familia y su aprobación o rechazo son definitivos en el rendimiento académico de los estudiantes. Por ende, los padres y familiares colindantes al estudiante deben inquietarse más por atender y comprender las actividades que el estudiante desarrolla en la escuela, por saber lo que le pasa en ella, quiénes son sus compañeros y amigos, y los intereses que originan sus vínculos, para implicarse en ellos cuando sea necesario.

De acuerdo con Jover y Sampascual (2008), el hecho de que el estudiante arremeta el estudio como un medio o un instrumento para lograr recompensas (querer tener en el futuro un buen trabajo o anhelar tener una buena posición social en el futuro), subyace a la meta instrumental de premio derivada de este trabajo. En suma, a modo de conclusión, de este estudio se desglosa que, aparte de las metas que revistan presentar los estudiantes videntes, se han manifestado otras dos (meta de superación de retos y meta instrumental de distinción) que podrían sujetar a las características del desarrollo de las personas ciegas o con deficiencia visual. Si bien 
este último extremo habría que examinarlo y contrastarlo con métodos más apropiados (tales como el análisis confirmatorio).

\section{Referencias bibliográficas}

Avendaño, H. (2013). El educando social: sujeto educativo del neoliberalismo [tesis de maestría]. Universidad Pedagógica Nacional, Bogotá, Colombia.

Barca, A., Peralbo, M., Porto, A., Marcos, J. y Brenilla, J. (2011). Metas académicas del alumnado de Educación Secundaria Obligatoria (ESO) y Bachillerato con alto y bajo rendimiento escolar. Revista de Educación, 354, 341-368.

Barón, F. J. y Téllez, F. (2004). Apuntes de bioestadística, Tercer ciclo de en ciencias de la Salud y Medicina. México: Universidad de Málaga.

Cepeda, E. y Caicedo, G. (2007). Factores asociados a la calidad de la educación. Revista Iberoamericana de Educación, 43(4), 1-7.

Cornejo, R. y Redondo, J. (2007). Variables y factores asociados al aprendizaje escolar. Una discusión desde la investigación actual. Estudios Pedagógicos, XXXIII(2), 155-175.

Dweck, C. S. (1986). Motivational processes affecting learning. American Psychologist, 41(10), 1040-1048.

Dweck, C. S. y Elliot, E. S. (1983). Achievement Motivation. En P. Mussen y E. M. Hetherington (eds.), Handbook of Child Psychology (pp. 643691). Nueva York: Wiley.

Elliot, A. J. y Harackiewicz, J. M. (1996). Approach and avoidance achievement goals and intrinsic motivation: A mediational analysis. Journal of Personality and Social Psychology, 70, 461-475. 
Jover, I. y Sampascual, G. (2008). Metas académicas en alumnos con ceguera y deficiencia visual. Revista Española de Pedagogía, 239, 49-64 Recuperado de https://dialnet.unirioja.es/descarga/articulo/2595609. pdf

Méndez, L. y Peña, J. (2007). Manual práctico para el diseño de la escala Likert. Recuperado de www.lasallep.edu.mx > Inicio , Vol 2, No 4 (2007) , Maldonado Luna

Minitab. (s. f). Interpretar todos los estadísticos y gráficas para 2 varianzas. Recuperado de https://support.minitab.com/es-mx/ minitab/18/help-and-how-to/statistics/basic-statistics/how-to/2variances/interpret-the-results/all-statistics-and-graphs/

Navarro, R. (2003). Factores asociados al rendimiento académico. Revista Iberoamericana de Educación, 33(1), 1-20.

Navas, L., Iborra, G. y Sampascual, G. (2007). Las metas académicas de los estudiantes de ESO en la clase de música. Revista de Psicodidáctica, 12(1), 131-142.

Lieury, A. y Fenouillet, F. (2006) Motivación y éxito escolar. México. Fce.

Piñeros, L. J. y Rodríguez, A. (1998) Los insumos escolares y en la educación secundaria y su efecto en el rendimiento académico de los estudiantes: un estudio en Colombia. Washington, D. C.: Banco Mundial.

Pérez, A. y Castejón, J. (1997) Factores que distinguen y explican el rendimiento académico diferencial de alumnos repetidores y alumnos cuya edad escolar coincide con su edad cronológica. Revista Psicodidáctica, 4, 103-117.

Redondo, J. (1997) La dinámica escolar: de la diferencia a la desigualdad. Revista de Psicología, 6, 7-18.

Rodríguez, L. y Avendaño, H. (2018). Análisis de los factores motivacionales de estudiantes de secundaria, en el planteamiento de una meta académica: una visión desde las Ciencias Naturales. Tecné, 
Episteme y Didaxis: VIII Congreso Internacional sobre Formación de Profesores de Ciencias (número extraordinario). Recuperado de https:// revistas.pedagogica.edu.co/index.php/TED/article/view/8885/6666

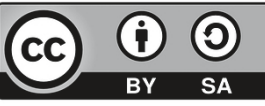

